\begin{abstract}
Iranica
Abstracta Iranica Revue bibliographique pour le domaine irano-aryen

Volume 34-35-36 | 2017

Comptes rendus des publications de 2011-2013
\end{abstract}

\title{
N. Sims-Williams. Differential Object Marking in Bactrian
}

\section{Agnès Lenepveu-Hotz}

\section{(2) OpenEdition}

10 Journals

\section{Édition électronique}

URL : http://journals.openedition.org/abstractairanica/39942

DOI : 10.4000/abstractairanica.39942

ISSN : 1961-960X

Éditeur :

CNRS (UMR 7528 Mondes iraniens et indiens), Éditions de l'IFRI

Référence électronique

Agnès Lenepveu-Hotz, « N. Sims-Williams. Differential Object Marking in Bactrian », Abstracta Iranica [En ligne], Volume 34-35-36 | 2017, document 1, mis en ligne le 15 juillet 2016, consulté le 03 octobre 2020. URL : http://journals.openedition.org/abstractairanica/39942 ; DOI : https://doi.org/10.4000/ abstractairanica.39942

Ce document a été généré automatiquement le 3 octobre 2020.

Tous droits réservés 


\title{
N. Sims-Williams. Differential Object Marking in Bactrian
}

\author{
Agnès Lenepveu-Hotz
}

\section{RÉFÉRENCE}

N. Sims-Williams. « Differential Object Marking in Bactrian », in: Agnes Korn, Geoffrey Haig, Simin Karimi, Pollet Samvelian, éds., Topics in Iranian Linguistics. Wiesbaden, Reichert, 2011, p. 23-38.

1 Dans cet article, l'A. traite du problème du marquage de l'objet en bactrien (langue du moyen-iranien, $\mathrm{I}^{\mathrm{er}}-\mathrm{IX}^{\mathrm{e}} \mathrm{s}$.) dans les phrases monotransitives (biactancielles) et ditransitives (triactancielles). Il montre dans un premier temps que le bactrien connaît une fracture d'actance entre le présent et le passé : le présent offre une structure accusative (l'agent est traité comme le sujet d'un verbe intransitif : il est au cas nominatif, et commande l'accord du verbe) et le passé, une structure ergative (le patient est traité comme le sujet d'un verbe intransitif, alors que l'agent est au cas oblique). C'est la situation que connaissent d'autres langues iraniennes. En revanche, là où le bactrien se démarque, c'est qu'il possède un marquage différentiel de l'objet aussi bien à l'accusatif qu'à l'ergatif : au passé également, situation que les langues iraniennes modernes ne présentent pas, le patient est introduit par la préposition av s'il s'agit d'une personne hautement individualisée. L'A. rapproche ce phénomène du moyen perse et $\mathrm{du}$ parthe où le patient est introduit par la préposition $\bar{o}$ (or l'étymologie de ces deux prépositions est la même, à savoir l'iranien ancien *abi).

2 Dans un deuxième temps, l'A. aborde le fonctionnement des verbes ditransitifs. Comme d'autres langues iraniennes, le bactrien possède un marquage différentiel de l'objet indirect, selon qu'il s'agit ou non d'une personne hautement individualisée. D'autre part, il existe des cas où le verbe au passé s'accorde avec ce que l'A. nomme R, c'est-àdire le destinataire de l'action (traditionnellement appelé objet indirect) : pour qu'il y 
ait un tel accord, l'objet direct ( $\mathrm{T}$ pour l'A.) est à la troisième personne et l'objet indirect $(\mathrm{R})$ est une première ou une deuxième personne du singulier.

3 L'A. finit sur des considérations diachroniques et typologiques. Nous rappelant que les textes bactriens ont été composés sur plus de sept siècles, l'A. retrace l'évolution du marquage de l'objet. En bactrien tardif (à partir de la fin du VII ${ }^{e}$ s.), le marquage différentiel de l'objet indirect disparaît ; dans les inscriptions de la période kouchane ( $\mathrm{I}^{\mathrm{er}}-\mathrm{III}{ }^{\mathrm{e}} \mathrm{s}$.), ce marquage différentiel n'existe pas encore. D'autres langues connaissent ces phénomènes de marquage différentiel, ils correspondent souvent à des cas de désambiguïsation. Ainsi en bactrien, une ambiguïté était possible quand $T$ et $R$ (objet direct et objet indirect) sont tous les deux des personnes. La préposition far a alors été utilisée pour $\mathrm{R}$ afin de le distinguer de $\mathrm{T}$, puis ce procédé de désambiguïsation a été généralisé à tous les cas où $\mathrm{R}$ était une personne.

\section{AUTEURS}

\section{AGNÈS LENEPVEU-HOTZ}

Université Sorbonne Nouvelle - Paris 3 